\title{
Qualidade do sono e prevalência das perturbações do sono em crianças saudáveis em Gaia: um estudo transversal
}

M ${ }^{\mathrm{a}}$ Adriana Rangel, ${ }^{1}$ Carolina Baptista, ${ }^{1}$ Ma João Pitta, ${ }^{2}$ Sara Anjo, ${ }^{2}$ Ana Luísa Leite ${ }^{3}$

\section{RESUMO}

Objetivos: Avaliar a qualidade do sono e estimar a prevalência das perturbações do sono em crianças dos dois aos 10 anos de idade num centro de saúde da área metropolitana do Porto.

Tipo de estudo: Estudo observacional descritivo e transversal.

Local: Consulta de saúde infantil e juvenil num centro de saúde na área urbana do ACeS Grande Porto VII - Gaia.

População: Crianças dos 2 aos 10 anos inscritas num centro de saúde do ACeS Grande PortoVII - Gaia.

Métodos: Aplicação do questionário Portuguese Children's Sleep Habits Questionnaire (CSHQ-PT), traduzido e validado para a população portuguesa, bem como um inquérito padronizado com questões individuais a crianças entre os 2-10 anos, observadas em Consulta de Saúde Infantil e Juvenil, de abril a junho de 2014.

Resultados: A amostra foi composta por 131 crianças com mediana de idades de cinco anos, 55\% em idade pré-escolar, com predomínio do sexo masculino (53,5\%). O índice de perturbação do sono (IPS) associou-se com o grupo etário (maior na idade pré-escolar, $p=0,032$ ) e, considerando o cut-off de $41,80,2 \%$ apresentaram um IPS elevado (mediana=47). Um IPS superior associou-se à necessidade de um familiar no quarto $(p=0,005)$ ou na cama $(p<0,001)$ para adormecer, visualização de televisão $(p=0,006)$ e uso de videojogos $(p=0,04)$. As crianças que partilhavam quarto com irmão ou dormiam sozinhas apresentaram um IPS inferior às que partilhavam quarto com os pais $(p=0,015)$. Um IPS inferior associou-se a um rendimento escolar superior $(p=0,029)$.

Conclusão: Os problemas comportamentais do sono são frequentes na nossa população, particularmente em idades mais jovens, existindo associação significativa com a necessidade da presença de um familiar no quarto ou na cama para adormecer, bem como à visualização de televisão e uso de videojogos, estando este dois últimos associados a um menor tempo total de sono.

Palavras-chave: Transtornos do Sono; Determinantes Epidemiológicos; Cuidados Primários de Saúde.

\section{INTRODUÇÃO}

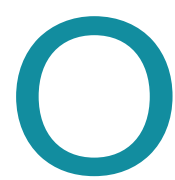

sono apresenta um papel vital no crescimento e desenvolvimento das crianças sendo, no entanto, as suas perturbações frequentemente subdiagnosticadas. ${ }^{1}$ É um processo fisiológico complexo, influenciado por propriedades biológicas intrínsecas, temperamento, expectativas, normas culturais e condições ambientais. ${ }^{2}$

Estudos demonstram que tanto os médicos como os pais não estão, em muitos casos, preparados para iden- tificar e abordar este tipo de problema nas consultas. ${ }^{3-}$ ${ }^{4}$ Estima-se que cerca de $25 \%$ das crianças tenham, nalguma fase da sua infância, uma perturbação do sono. ${ }^{5}$ Em Portugal, os dados disponíveis sobre prevalência das perturbações do sono são consideravelmente me-

'Médica Interna de Pediatria, Serviço de Pediatria, Centro Hospitalar de Vila Nova de Gaia e Espinho, EPE.

2Médica Interna de Medicina Geral e Familiar, UCSP Barão do Corvo, ACeS Grande Porto VII - Gaia.

${ }^{3}$ Médica Assistente Hospitalar de Pediatria, Serviço de Pediatria, Centro Hospitalar de Vila Nova de Gaia e Espinho, EPE. 
nores quando comparados com a literatura internacional, variando de 5,6 a 32\%.6.8 Apesar de frequentes, as alterações do sono são culturalmente aceites por uma grande parte dos pais e, dessa forma, subvalorizados. ${ }^{6}$

Sabe-se que as perturbações do sono podem causar morbilidade substancial na criança, nomeadamente consequências a nível do comportamento, da aprendizagem e mesmo perturbações de desenvolvimento como hiperatividade, défice de atenção, défice cognitivo, depressão, risco de quedas acidentais e obesidade. ${ }^{2,9-10}$ Além das complicações mencionadas nas crianças, estas perturbações podem também ocasionar nos pais privação de sono, fadiga, alterações do humor, frustração, afetando o desempenho parental e constituindo até fator de risco para violência familiar e divórcio. ${ }^{2,5}$

A abordagem dos hábitos de sono, quer pela família quer pelo médico, nem sempre é feita nas consultas de vigilância de saúde infantil e juvenil, ficando a deteção de possíveis perturbações para uma fase mais tardia em que as manifestações e suas repercussões já se fazem sentir. ${ }^{5}$ Por este motivo, torna-se fulcral a abordagem sistemática deste tema nas consultas de saúde infantil como forma de prevenção, ${ }^{2}$ dado que a instituição de regras para uma boa higiene de sono pode ser suficiente para corrigir algumas destas perturbações. ${ }^{5}$

Embora seja cada vez mais reconhecida a importância do sono na saúde infantil e juvenil, existem ainda poucos estudos sobre esta questão em Portugal baseados em inquéritos padronizados. Recentemente elaborado, traduzido e devidamente validado para a população portuguesa, o Portuguese Children's Sleep Habits Questionnaire-CSHQ-PT tem por objetivo avaliar os hábitos de sono e detetar eventuais problemas associados. ${ }^{11}$ Em Portugal foi aplicado por Silva e colaboradores a uma população escolar da região metropolitana de Lisboa. ${ }^{6}$ Deste modo, o objetivo principal do presente estudo foi avaliar a qualidade do sono e estimar a prevalência das perturbações do sono em crianças dos dois aos 10 anos de idade num centro de saúde da área metropolitana do Porto através da aplicação do CSHQ-PT. Como objetivos complementares comparam-se estes achados com o estudo referido ${ }^{6}$ e estudaram-se fatores individuais, sociais e familiares, com influência na qualidade do sono.

\section{MATERIAL E MÉTODOS}

Elaborou-se um estudo observacional descritivo e transversal. A amostra foi constituída por crianças com idades entre os dois e os 10 anos, observadas em consulta de saúde infantil e juvenil, num centro de saúde da área urbana do ACeS Grande Porto VII - Gaia, no período de abril a junho de 2014. A amostra foi dividida em dois grupos consoante a idade: pré-escolar (dois aos cinco anos de idade) e escolar (seis aos 10 anos de idade).

Previamente estimou-se como universo amostral representativo necessário um total de cerca de 90 questionários, considerando um erro amostral de $5 \%$, com intervalo de confiança 95\% e numa população de 18.985 crianças em idade pré-escolar e escolar (números do Ministério da Educação), com uma prevalência mínima esperada de $5,6 \%$.

Os critérios de inclusão foram a idade elegível da criança e a vontade dos pais em participar com consentimento informado e escrito. A cada caso elegível foi aplicado, e respondido presencialmente na consulta, $o$ CSHQ-PT ao acompanhante da criança à CSIJ. Este questionário foi complementado com um inquérito padronizado sobre fatores sociofamiliares e individuais elaborado pelos autores e testado previamente num período experimental.

A versão portuguesa do CSHQ-PT foi validada para crianças dos dois aos 10 anos de idade e encontra-se disponível online. ${ }^{11} \mathrm{O}$ questionário permite avaliar as horas de acordar e de deitar (à semana e ao fim-de-semana), bem como a duração habitual do sono e a frequência de diversos comportamentos relacionados com o sono.

Alguns comportamentos-problema, que correspondem ao mesmo tipo de distúrbio do sono, podem ser agrupados em subescalas (tabela I).

Realizou-se a soma da cotação total das questões para obtenção do Índice de Perturbação do Sono (IPS) e a soma parcelar nas diferentes subescalas (tabela I).

O questionário anexado ao CSHQ-PT, elaborado pelos autores, incluiu questões sobre antecedentes patológicos e medicação diária da criança, rendimento escolar, prática de exercício físico, bem como hábitos e rituais antes de adormecer, entre outras. Incluiu ainda questões sobre o tipo de agregado familiar, escolaridade e profissão dos pais (incluindo trabalho por turnos 


\begin{tabular}{|c|c|}
\hline Subescala & Questões \\
\hline Subescala 1 - Resistência em ir para a cama & $1,3,4,5,6,8$ \\
\hline Subescala 2 - Início do sono & 2 \\
\hline Subescala 3 - Duração do sono & $9,10,11$ \\
\hline Subescala 4 - Ansiedade associada ao sono & $5,7,8,21$ \\
\hline Subescala 5 - Despertares noturnos & $16,24,25$ \\
\hline Subescala 6 - Parassónias & $12,13,14,15,17,22,23$ \\
\hline Subescala 7 - Perturbações respiratórias do sono & $18,19,20$ \\
\hline Subescala 8 - Sonolência diurna & $26,27,28,29,30,31,32,33$ \\
\hline
\end{tabular}

fantário e/ou pré-escola. A mediana da idade de entrada no infantário foi 24 (AIQ: 12-36) meses.

A restante caracterização sociodemográfica da amostra é resumida na tabela II.

Relativamente à constituição do agregado familiar, na maioria dos domicílios reside uma mediana de quatro pessoas ( $\min =2$; máx $=8$ ), sendo a maioria dos agregados do tipo nuclear $(77,7 \%)$. No que diz respeito ao número de irmãos, a mediana é um ( $\min =0$; máx=4). A maioria das habitações tem dois (min=1;

e trabalho noturno), antecedentes de insónia, depressão, necessidade de medicação e hábitos parentais para adormecer.

Na análise estatística utilizou-se a versão 21.0 do programa SPSS $®($ IBM $®$, SPSS $®$ Statistics Inc., Chicago). O teste de Shapiro-Wilk $(p>0,05)$ foi usado para testar a normalidade das variáveis quantitativas. A comparação de frequências relativas, de distribuições e de valores médios foi avaliado através de testes de qui-quadrado (variáveis categóricas), Mann-Whitneye Kruskal Wallis (variáveis numéricas de distribuição não normal) e testes $t$ de student e ANOVA (variáveis numéricas paramétricas). Considerou-se uma probabilidade de erro tipo I $(\alpha)$ de 0,05 .

O estudo foi aprovado pela Comissão de Ética da Administração Regional de Saúde (ARS) do Norte e foi concedida autorização dos autores da versão portuguesa do CSHQ-PT para aplicação do mesmo neste estudo.

\section{RESULTADOS}

Foram respondidos um total de 141 inquéritos, dos quais $10(7,1 \%)$ foram excluídos por preenchimento inválido ( $n=1$, idade superior a 10 anos) ou muito incompleto ( $n=9$, mais de $20 \%$ das respostas omissas). Deste modo, foram incluídos no estudo dados referentes a 131 crianças.

Destas 131 crianças, a idade mediana correspondeu aos cinco (Amplitude Interquartil-AIQ: 3-7) anos, com predomínio do sexo masculino ( $n=70,53,5 \%)$. Cinquenta e cinco por cento $(n=72)$ correspondiam a crianças em idade pré-escolar (dois aos cinco anos de idade), das quais apenas nove ainda não frequentavam in- máx=4) quartos $(49,6 \%)$ e, na maioria dos casos, a criança partilha-o com um irmão $(42,6 \%)$ ou não o partilha com ninguém $(41,9 \%)$. A idade mediana de saída do quarto dos pais é o ano de idade ( $\mathrm{min}=0$; máx=6); contudo, 13,2\% da amostra ainda partilhava quarto com os pais $\left(M_{d}\right.$ da idade três anos; AIQ: 2-7).

\section{Hábitos e rituais para adormecer}

A mãe é quem deita a criança na maioria dos casos $(50,8 \%, n=66)$.

Em apenas 17,6\% ( $n=23$ ) os pais negam algum hábito ou ritual para a criança adormecer. Vinte e seis por cento necessitam de luz de presença, $22,1 \%$ necessitam de um familiar na cama para adormecer e $15,1 \%$ necessita de um familiar presente no quarto. $\mathrm{O}$ uso de chupeta foi reportado em $21,5 \%$ ( $n=28$ ), sendo significativamente mais frequente na idade pré-escolar $(p=0,001)$.

Quanto aos rituais antes de adormecer, $47,3 \%$ das crianças bebem leite, sendo significativamente mais frequente na idade pré-escolar $(p=0,008) ; 36,6 \%$ leem e/ou ouvem uma história e 35,9\% veem televisão. O uso de videojogos foi referido em apenas dois casos (1,5\%).

A realização de sesta diária é mais frequente na idade pré-escolar $(48,6 \% v s .5,1 \%, p<0,001)$ e a proporção de crianças que dorme sesta nos diferentes grupos etários decresce com o aumento da idade.

A frequência dos diferentes hábitos e rituais assinalados no inquérito encontram-se ilustrados na tabela III, bem como a comparação entre grupos (escolar $v s$. pré-escolar). 


\begin{tabular}{|c|c|c|}
\hline $\begin{array}{l}\text { QUADRO II. Caracterização sociodemogrt } \\
\text { amostra }(n=131)\end{array}$ & & \\
\hline Variável & $\mathbf{N}$ & $\%$ \\
\hline Seguimento em Pediatra Particular & 27 & $9,2 \%$ \\
\hline Seguimento em Consulta Hospitalar & 29 & $22,1 \%$ \\
\hline Antecedentes de Prematuridade & 9 & $6,9 \%$ \\
\hline Antecedentes patológicos & 16 & $12,2 \%$ \\
\hline Patologia atópica (asma, rinite alérgica, APLV) & 6 & $4,58 \%$ \\
\hline $\begin{array}{l}\text { Patologia do neurodesenvolvimento (PHDA, } \\
\text { dislexia, atraso cognitivo, atraso da linguagem) }\end{array}$ & 6 & $4,58 \%$ \\
\hline Anemia ferripriva & 1 & $0,8 \%$ \\
\hline Doença de Legg-Perths & 1 & $0,8 \%$ \\
\hline Missings & 2 & $1,53 \%$ \\
\hline Medicação crónica & 12 & $9,2 \%$ \\
\hline Metilfenidato & 3 & $2,29 \%$ \\
\hline Cetirizina & 1 & $0,8 \%$ \\
\hline Montelucaste & 1 & $0,8 \%$ \\
\hline Corticoide e broncodilatador inalado & 1 & $0,8 \%$ \\
\hline Ferro (PO) & 1 & $0,8 \%$ \\
\hline Valproato de sódio & 1 & $0,8 \%$ \\
\hline Missings & 4 & $3,05 \%$ \\
\hline \multicolumn{3}{|l|}{ Escolaridade da mãe } \\
\hline$<4$. $^{\text {a }}$ classe & 2 & $1,7 \%$ \\
\hline 4. ${ }^{\mathrm{a}}$ classe $-8 .^{\circ}$ ano & 20 & $16,5 \%$ \\
\hline $9 .^{\circ}$ ano $-11 .^{\circ}$ ano & 36 & $29,8 \%$ \\
\hline $12 .^{\circ}$ ano & 32 & $26,4 \%$ \\
\hline Licenciatura/Bacharelato ou superior & 31 & $25,6 \%$ \\
\hline \multicolumn{3}{|l|}{ Escolaridade do pai } \\
\hline$<4$. $^{\text {a classe }}$ & 2 & $1,7 \%$ \\
\hline $4 .^{\mathrm{a}}$ classe $-8 .^{\circ}$ ano & 30 & $25,2 \%$ \\
\hline $9 .^{\circ}$ ano $-11 .^{\circ}$ ano & 34 & $28,6 \%$ \\
\hline $12 .^{\circ}$ ano & 24 & 20,2 \\
\hline Licenciatura/Bacharelato ou superior & 29 & $24,3 \%$ \\
\hline \multicolumn{3}{|l|}{ Situação profissional } \\
\hline Ambos empregados & 59 & $47,2 \%$ \\
\hline Pelo menos um empregado & 50 & $40,0 \%$ \\
\hline Ambos desempregados & 16 & $12,8 \%$ \\
\hline Missings & 6 & $4,6 \%$ \\
\hline
\end{tabular}

\section{Qualidade do sono e problemas associados (CSHQ-PT)}

A mediana da hora de deitar nos dias de semana foi 21h30min (AIQ: $21 \mathrm{~h} 07$-22h00) e ao fim-de-semana às 22h00, não havendo diferenças estatisticamente significativas entre os grupos. A mediana da hora de acordar durante a semana foi 8h00 (AIQ: 7h30-8h15) e ao fim-de-semana 9h00 (AIQ: 8h00-10h00), ocorrendo mais cedo nas crianças em idade escolar $(p=0,051)$.

A mediana do tempo total de sono foi 10h00 (AIQ: 9h30-11h00), sendo superior na idade pré-escolar $(p=0,002)$ e decrescendo com o aumento da idade (figura 1). Quanto ao número mínimo de horas de sono, $56,3 \%$ e $41,1 \%$ das crianças no grupo pré-escolar e escolar, respetivamente, não dorme o número mínimo de horas recomendado (recomendações da Centersfor Disease Control and Prevention-disponível em www.cdc.gov/sleep/ about_sleep/how_much_sleep.htm).

O índice de perturbação do sono (IPS, cotação total do CSHQ-PT) na amostra tem uma mediana de 47 (AIQ: 42-51), que não difere significativamente do valor IPS encontrado por Silva e colaboradores ${ }^{6}$ na população da área metropolitana de Lisboa $(p=0,81)$. Considerando o cut off de $41,80,2 \%$ ( $n=105)$ apresenta um IPS elevado, decrescendo para 49,6\% ( $n=65$ ) quando considerado o cut off ajustado para a população portuguesa (IPS $>48$ ), sugerido por Silva e colaboradores. ${ }^{6}$

O IPS associou-se ao grupo etário, sendo mais elevado nas crianças em idade pré-escolar $(p=0,032)$. Na análise de subescalas do questionário, as crianças em idade pré-escolar apresentam cotações mais elevadas nas subescalas Resistência em ir para a cama $(p=0,001)$ e Parassónias $(p=0,024)$, não havendo diferenças estatisticamente significativas nas restantes subescalas (conforme descriminado na tabela IV).

O IPS não teve relação estatística com o género, antecedentes de prematuridade, presença de antecedente patológico reportado pelos pais ou com o uso diário de medicação.

Um IPS significativamente mais elevado associou-se à necessidade da presença de um familiar no quarto $(p=0,005)$ ou na cama $(p<0,001)$ para adormecer, bem como à visualização de televisão $(p=0,006)$ e uso de videojogos $(p=0,047)$, estando estes dois últimos associados a um menor tempo total de sono $(p<0,02)$. Não se verificou associação estatística com o uso de chupe- 


\begin{tabular}{|c|c|c|c|c|c|}
\hline \multicolumn{2}{|c|}{ Variável } & \multirow{2}{*}{$\begin{array}{c}\text { Total }(n=131) \\
28(21,4 \%)\end{array}$} & \multirow{2}{*}{$\begin{array}{c}\text { Pré-escolar }(\boldsymbol{n}=\mathbf{7 2}) \\
23(31,9 \%)\end{array}$} & \multirow{2}{*}{$\begin{array}{c}\text { Escolar }(n=59) \\
5(8,5 \%)\end{array}$} & \multirow{2}{*}{$\begin{array}{c}P \\
0,001\end{array}$} \\
\hline \multirow{6}{*}{ 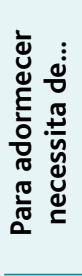 } & Chupeta & & & & \\
\hline & Fralda de pano ou cobertor & $11(8,4 \%)$ & $6(8,3 \%)$ & $5(8,5 \%)$ & NS \\
\hline & Luz de presença & $34(26,0 \%)$ & $19(26,4 \%)$ & $15(25,4 \%)$ & NS \\
\hline & Peluche ou boneco & $28(21,4 \%)$ & $16(22,2 \%)$ & $12(20,3 \%)$ & NS \\
\hline & Presença de um familiar na cama & $29(22,1 \%)$ & $20(27,8 \%)$ & $9(15,3 \%)$ & NS \\
\hline & Presença de um familiar no quarto & $20(15,3 \%)$ & $13(18,1 \%)$ & $7(11,9 \%)$ & NS \\
\hline \multirow{6}{*}{ 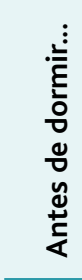 } & Lava os dentes & $109(83,2 \%)$ & $60(80,3 \%)$ & $49(83,1 \%)$ & NS \\
\hline & Toma banho & $44(33,6)$ & $21(29,2 \%)$ & $23(39,0 \%)$ & NS \\
\hline & Bebe leite & $62(47,3 \%)$ & $42(58,3 \%)$ & $20(33,9 \%)$ & 0,008 \\
\hline & Lê/ouve uma história & $48(36,6 \%)$ & $26(36,1 \%)$ & $22(37,3 \%)$ & NS \\
\hline & Vê televisão & $47(35,9 \%)$ & $23(31,9 \%)$ & $24(49,7 \%)$ & NS \\
\hline & Joga videojogos & $2(1,5 \%)$ & $1(1,4 \%)$ & $1(1,7 \%)$ & NS \\
\hline \multicolumn{2}{|c|}{ Faz sesta diariamente } & $38(29,0 \%)$ & $35(48,6 \%)$ & $3(5,1 \%)$ & $<0,001$ \\
\hline
\end{tabular}

ta ou objeto de transição, nem com a necessidade de luz de presença. As rotinas de higiene (lavar os dentes, tomar banho) ou o hábito de leitura antes de dormir também não mostraram relação com o IPS.

As crianças que partilhavam quarto com irmã(o) ou que dormiam sozinhas apresentaram um IPS significativamente menor que aquelas crianças que partilhavam quarto com os pais $(p=0,028)$. Um IPS inferior verificou-se também naquelas crianças que se deitavam sozinhas, ou que eram deitadas por ambos os pais, contrariamente aquelas que eram deitadas pela mãe ou pelo pai $(p=0,005)$.

No que concerne aos fatores sociais e familiares, não se obteve associação do IPS com o tipo de agregado familiar, número de pessoas a residir no domicílio, escolaridade dos pais ou a sua situação profissional, nomeadamente com o trabalho por turnos ou com turnos noturnos. Houve apenas relação entre o IPS e antecedentes de insónia reportado pelos pais $(p=0,033)$.

Um IPS inferior esteve estatisticamente associado a um rendimento escolar superior $(p=0,029)$. Não se obteve associação entre o IPS e a frequência de birras, queixas de distração ou impulsividade reportadas pelos pais. A prática de exercício físico não mostrou associação com IPS; contudo, associou-se a uma cotação inferior na subescala "despertares noturnos" $(p=0,032)$.

\section{DISCUSSÃO}

A infância é caracterizada por mudanças consideráveis na organização, duração e estrutura do sono, com elevada incidência de problemas de sono transitórios como resistência em ir para a cama e ansiedade relacionada com o sono. ${ }^{10} \mathrm{O}$ que é com frequência menos reconhecido são os problemas de sono crónicos que afetam até cerca de $30 \%$ das crianças. Além dos efeitos nefastos no neuro desenvolvimento da criança, os problemas de sono causam efeitos negativos a nível familiar. $^{5}$

A maioria dos estudos sobre o sono nas crianças portuguesas aponta para uma elevada frequência de problemas do sono e comportamentos deletérios na nossa população, utilizando, contudo, inquéritos não padronizados, dificultando a comparação. ${ }^{2,5,7,12}$ O CSHQ é um dos inquéritos padronizados mais usados mundialmente e foi recentemente validade e traduzido para a população portuguesa. ${ }^{11}$ Foi aplicado por Silva e colaboradores ${ }^{6}$ numa amostra significativa de crianças na área metropolitana de Lisboa e, à semelhança de outros estudos, aponta para uma elevada prevalência de perturbações do sono, sublinhando a necessidade de rastrear as mesmas. O valor médio do Índice de Perturbação do Sono (IPS) encontrado neste estudo $(46,51 \pm 6,80)$ não difere significativamente do encontrado no nosso. 


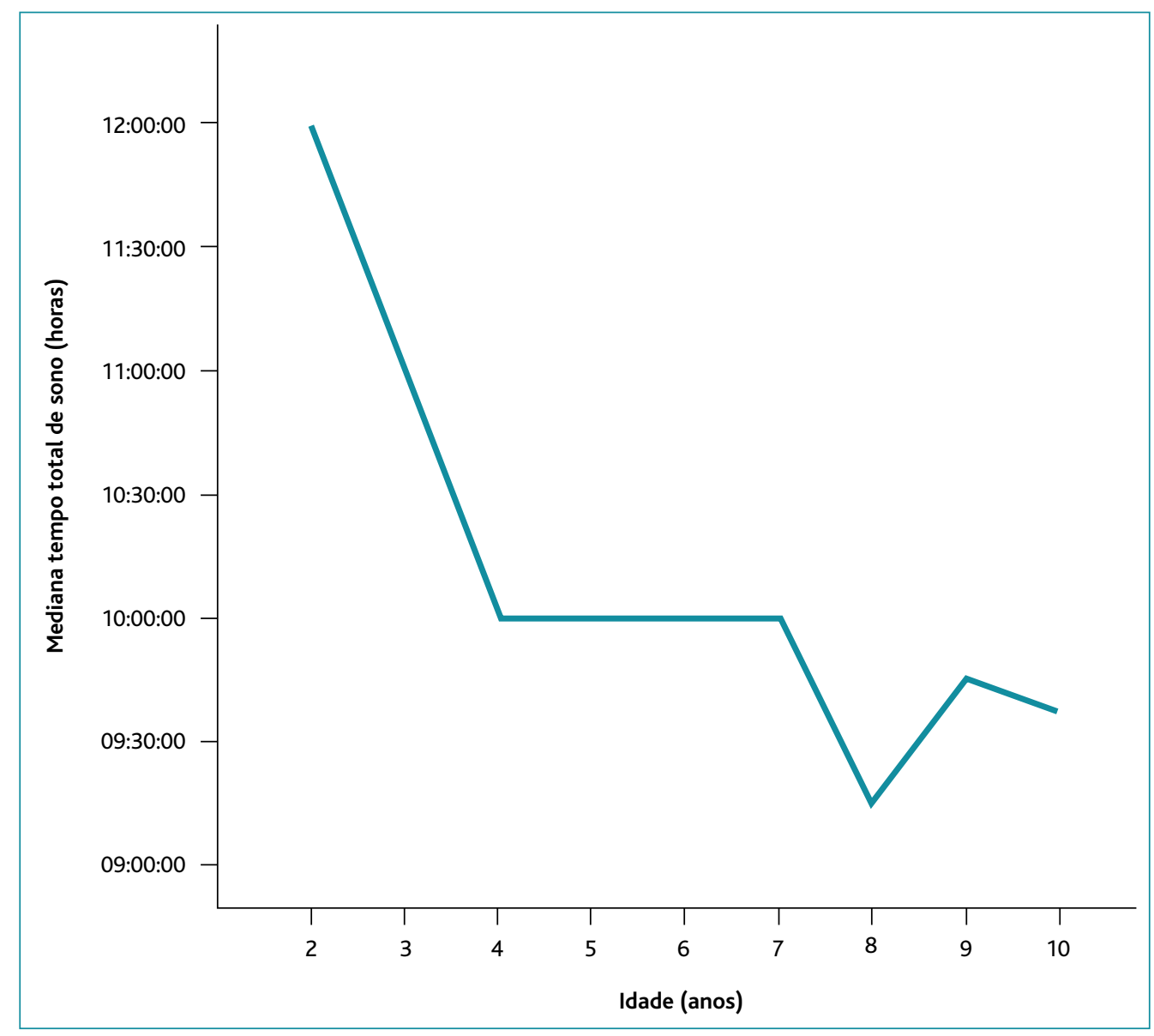

Figura 1. Mediana do tempo total de sono consoante a idade da criança.

em ir para a cama e parassónias. Este achado traduz a já conhecida maior prevalência das perturbações do início e manutenção do sono mais frequentes na criança pequena, bem como pesadelos, terrores noturnos e sonambulismo igualmente mais frequentes nesta idade. ${ }^{12}$ Deste modo, a inclusão de crianças em idade pré-escolar neste estudo pode ser responsável pela elevada proporção de crianças com IPS elevado.

Na presente análise detetaram-se alguns fatores estatisticamente associados a um IPS superior, nomeadamente a visualização de televi-

Na presente amostra, tendo em conta o cut off original de 41 para o Índice de Perturbação do Sono (IPS), $80,2 \%$ apresentavam um IPS elevado. Silva e colaboradores $^{6}$ sugerem o ajuste do cut offdo IPS de 41 para 48 , considerando este valor mais adequado à população portuguesa. Mesmo considerando este cut off, 49,6\% apresenta um IPS elevado, demonstrando a elevada prevalência de comportamentos problema no que concerne ao sono na população estudada. Contudo, não se sabe se é apenas uma questão cultural ou se, de facto, as crianças portuguesas têm uma prevalência superior de problemas de sono e desconhece-se qual a repercussão das mesmas.

O grupo pré-escolar apresentou um IPS significativamente superior, o que traduz uma maior frequência de comportamentos problema na criança mais jovem, particularmente neste caso nas subescalas resistência são e uso de videojogos, partilha de quarto com os pais, a necessidade de adormecer com um familiar presente no quarto ou na cama e história parental de insónia.

O efeito nefasto do tempo de ecrã foi já demonstrado, ${ }^{13-14}$ considerando-se que se deve a um atraso no início do sono.

Relativamente a estudos prévios, verifica-se que uma menor proporção de crianças via televisão antes de dormir (35,9\% vs. 71-77\%). ${ }^{5,7}$ Contudo, não foi questionado o uso de outros dispositivos eletrónicos, como tablets, telemóveis, etc., cuja utilização pode ter aumentado nos últimos anos.

A associação entre um IPS mais elevado e a necessidade de um familiar no quarto ou na cama para adormecer, contrapondo-se ao IPS inferior nas crianças que se deitam sozinhas e que partilham quarto com irmão, é consistente com o conceito de que o adormecimento 


\begin{tabular}{|c|c|c|c|c|c|}
\hline \multicolumn{6}{|c|}{$\begin{array}{l}\text { QUADRO IV. Índice de Perturbação do Sono e cotação nas subescalas - comparação } \\
\text { entre os grupos pré-escolar e escolar }\end{array}$} \\
\hline \multicolumn{2}{|c|}{ Variável } & $\begin{array}{c}\text { Total } \\
(n=131)^{*}\end{array}$ & $\begin{array}{l}\text { Pré-escolar } \\
(n=72)^{*}\end{array}$ & $\begin{array}{l}\text { Escolar } \\
(n=59)^{*}\end{array}$ & $P^{+}$ \\
\hline \multicolumn{2}{|c|}{ Índice de Perturbação do Sono (IPS) } & $47(42-51)$ & $48(43-51)$ & $43(40-51)$ & 0,032 \\
\hline \multirow{8}{*}{ 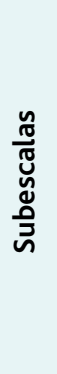 } & Resistência a ir para a cama & $8(6-11)$ & $9(7-10)$ & $7(6-10)$ & 0,001 \\
\hline & Início do sono & $2(1-3)$ & $2(1-2)$ & $2(1-3)$ & NS \\
\hline & Duração do sono & $3(3-4)$ & $4(3-4)$ & $3(3-4)$ & NS \\
\hline & Ansiedade associada ao sono & $6(4-7)$ & $6(4-8)$ & $5(4-6)$ & NS \\
\hline & Despertares noturnos & $4(3-5)$ & $4(3-5)$ & $3(3-5)$ & NS \\
\hline & Parassónias & $10(8-11)$ & $10(9-11)$ & $9(8-11)$ & 0,024 \\
\hline & Perturbação respiratória do sono & $3(3-4)$ & $3(3-4)$ & $3(3-4)$ & NS \\
\hline & Sonolência diurna & $12(10-14)$ & $12(10-14)$ & $12(10-14)$ & NS \\
\hline
\end{tabular}

*Mediana (amplitude interquartil); + Mann-Whitney

independente, em cama própria, é favorecedor de uma melhor qualidade de sono, conferindo à criança a capacidade de readormecer sozinha perante um despertar noturno.

O co-sleeping, isto é, a partilha da cama dos pais, é um comportamento desaprovado pela maioria das sociedades ocidentais por considerarem que não promove o adormecimento autónomo e, por outro lado, porque diminui a qualidade do sono, estando associado a maior sonolência diurna. ${ }^{15}$ Todavia, convém salientar que o sono é fortemente moldado por valores culturais, crenças e expectativas dos pais. ${ }^{16}$ As normas culturais determinam os limites entre os comportamentos de sono "normais" e "problemáticos". Várias culturas não só promovem o co-sleeping como consideram que o quarto próprio para a criança é prejudicial. ${ }^{16}$ É por isso fundamental compreender o que constituiu de facto um problema de sono, quando, a quem e a melhor forma de abordar. Muitas vezes a abordagem envolve a modificação ou introdução de novos hábitos por forma a equilibrar as necessidades da criança e dos pais.

Os objetos de transição, normalmente objetos como a chupeta, a fralda, o peluche, facilitam o adormecimento ao providenciar uma sensação de conforto e segurança à criança, podendo ser usados com o objetivo de promover o adormecimento independente, ${ }^{16}$ não tendo demonstrado um efeito negativo no IPS. A associação entre um IPS superior e a história familiar de in- sónia denota a influência genética na estrutura do sono, embora menor que os fatores ambientais. ${ }^{17-18}$

A duração adequada do sono é um aspeto com frequência negligenciado. A redução do número de horas de sono pode ter os mesmos efeitos negativos que as restantes perturbações do sono. A este respeito verifica-se na presente amostra que a duração total do sono (DTS), à semelhança de outros estudos, ${ }^{19-21}$ decresce ao longo da idade (figura 1), de uma mediana de 12h00 (AIQ: 2h45) aos dois anos, para $9 \mathrm{~h} 37$ (AIQ: 1h) aos 10 anos. A depressão na DTS aos oito anos de idade ( $M_{d}=9$ h15; AIQ 1h) não apresenta explicação aparente, podendo ser consequência do reduzido número do grupo ( $n=8)$, com dispersão de valores elevada. O decréscimo na DTS ocorreu de forma paralela a uma diminuição da proporção de crianças que faz sesta, sendo que apenas três crianças no grupo escolar $(5,1 \%)$ realizam sesta diariamente. Obteve-se uma mediana de DTS que foi superior à média em Espanha, ${ }^{22}$ mas inferior a outros países europeus, nomeadamente Inglaterra, Irlanda, Suíça. ${ }^{20-21,23} \mathrm{O}$ tempo total de sono diário correlaciona-se inversamente com a idade, bem como com a hora de deitar mais tardia, ${ }^{13-14,19}$ uma tendência que se tem vindo a agravar nos últimos anos, conforme demonstrado por Iglowstein e colaboradores. ${ }^{21}$

Na presente amostra, uma percentagem significativa de crianças (41,1-56,3\%) não dorme o número mínimo de horas recomendado. Esta percentagem pode ainda ser mais importante, uma vez que o tempo de sono reportado pelos pais é habitualmente ligeiramente superior ao tempo de sono real, traduzindo mais o "tempo na cama" do que propriamente o tempo a dormir. Sendo a DTS fortemente determinada por fatores ambientais ( 60\%), há uma importante janela de oportunidade de intervenção. ${ }^{14,16}$

É fundamental salientar que os problemas de sono são com frequência subvalorizados e subdiagnosticados. Este facto deve-se, por um lado, ao facto de os pro- 
fissionais de saúde com frequência não questionarem sobre o tema nas CSIJ, pressupondo que os pais espontaneamente levantarão essa questão., ${ }^{3,24}$ Contudo, o conhecimento dos pais acerca dos normais padrões de sono ao longo da infância, eventuais problemas e duração adequada do sono é claramente insuficiente. ${ }^{4}$ Este facto, aliado ao desconhecimento populacional dos efeitos negativos que estes exercem, leva a que estes sejam subreportados pelos pais nas CSIJ. ${ }^{10}$ De facto, nos estudos realizados em Portugal, apesar de uma percentagem significativa considerar que o seu filho tinha um problema de sono (74-84\%), o tema só tinha sido abordado como queixa na CSIJ em $7-13 \%{ }^{2,7}$

Como limitações deste trabalho, refiram-se as inerentes ao facto de se tratar de um estudo observacional, ao processo e dimensão amostral. Por ter sido realizado num único centro de saúde deVila Nova de Gaia, o que limitou a heterogeneidade geográfica e populacional, não possibilita, obviamente, a generalização à população de Vila Nova de Gaia. Contudo, o presente estudo permite salientar, ao identificar uma elevada prevalência de comportamentos problema nesta população, a necessidade de realização de estudos futuros nesta área, nomeadamente estudos que avaliem as repercussões destes comportamentos a nível familiar e na criança (comportamento, rendimento escolar, etc.).

Uma vez que não foi questionado ao acompanhante da criança a sua perceção da qualidade do sono da mesma ou se este considerava que a criança tinha algum problema de sono, não foi possível estimar o grau de concordância entre esta avaliação subjetiva e o IPS obtido - um dado que poderia ser interessante. Também não foi questionado se o tema já tinha sido abordado com o médico assistente/pediatra que acompanha normalmente a criança.

O preenchimento presencial do inquérito pode ter causado algum viés de resposta em algumas questões (nomeadamente quanto aos rituais antes de dormir), o que poderia explicar, por exemplo, a redução significativa da percentagem de crianças que via televisão antes de deitar face a estudos anteriores.

Uma vez que os antecedentes patológicos e medicação foram assinalados pelo acompanhante, uma percentagem destes poderá não ter sido referida e, portanto, ser superior ao estimado. Pelo facto de algumas patologias e medicação crónicas poderem influenciar negativamente a qualidade do sono, as mesmas poderiam explicar a elevada prevalência de perturbações do sono encontrada.

\section{AGRADECIMENTOS}

Um agradecimento especial à $\mathrm{Dr}^{\mathrm{a}}$ Isabel Ribeiro, minha orientadora de formação no estágio de Cuidados de Saúde Primários à Criança e ao Adolescente, que muito me ajudou na concretização deste trabalho.

\section{REFERÊNCIAS BIBLIOGRÁFICAS}

1. Bhargava S. Diagnosis and management of common sleep problems in children. Pediatr Rev. 2011;32(3):91-8.

2. Mendes LR, Fernandes A, Garcia FT. Hábitos e perturbações do sono em crianças em idade escolar [Sleep habits and sleep problems in school aged children]. Acta Pediatr Port 2004;35(4):341-7. Portuguese

3. Owens JA. The practice of pediatric sleep medicine: results of a community survey. Pediatrics. 2001;108(3):E51.

4. Schreck KA, Richdale AL. Knowledge of childhood sleep: a possible variable in under or misdiagnosis of childhood sleep problems. J Sleep Res. 2011;20(4):589-97.

5. Morais S, Veiga Z, Estevão MH. Hábitos e perturbações do sono numa população Pediátrica de Coimbra [Sleep habits and sleep problems in a pediatric population in Coimbra]. Saúde Infantil. 2007;29(1):15-22. Portuguese

6. Silva FG, Silva CR, Braga LB, Neto AS. Hábitos e problemas do sono dos dois aos dez anos: estudo populacional [Sleep habits and sleep problems in Portuguese children from two to ten years old: a population-based study]. Acta Pediatr Port. 2013;44(5):196-202. Portuguese

7. Pedrosa C, Cruz G, Pereira SA. Hábitos e perturbações do sono de uma população infantil de Vila Nova de Gaia [Sleep practices and disorders in a paediatric population of Vila Nova de Gaia]. Acta Pediatr Port. 2004;35(4):323-8. Portuguese

8. Klein JM, Gonçalves A. Problemas de sono-vigilia em criancas: um estudo da prevalencia [Sleep-wake problems in children: a study about prevalence]. Psico-USF. 2008;13(1):51-8. Portuguese

9. Astill RG, Van der Heijden KB, Van IJzendoorn MH, Van Someren EJ. Sleep, cognition, and behavioral problems in school age children: a century of research meta-analyzed. Psychol Bull. 2012;138(6):1109-38.

10. Blunden S, Lushington K, Lorenzen B, Ooi T, Fung F, Kennedy D. Are sleep problems under-recognised in general practice? Arch Dis Child. 2004;89(8):708-12.

11. Silva FG, Silva CR, Braga LB, Neto AS. Portuguese Children's Sleep Habits Questionnaire: validation and cross-cultural comparison. J Pediatr (Rio J). 2014;90(1):78-84

12. Duarte C, Santos I, Estêvão MH. Perturbações do sono na criança [Sleep disorders in children]. Acta Pediatr Port 2004;35(4):349-57. Portuguese

13. Foley LS, Maddison R, Jiang Y, Marsh S, Olds T, Ridley K. Presleep activities and time of sleep onset in children. Pediatrics. 2013;131(2):27682.

14. Nixon GM, Thompson JM, Han DY, Becroft DM, Clark PM, Robinson E, et al. Short sleep duration in middle childhood: risk factors and consequences. Sleep. 2008;31(1):71-8. 
15. Liu X, Liu L, Wang R. Bed sharing, sleep habits, and sleep problems among Chinese school-aged children. Sleep. 2003;26(7):839-44.

16. Jenni OG, O'Connor BB. Children's sleep: an interplay between culture and biology. Pediatrics. 2005;115(1 Suppl):204-16.

17. Fisher $\mathrm{A}$, van Jaarsveld $\mathrm{CH}$, Llewellyn $\mathrm{CH}$, Wardle J. Genetic and environmental influences on infant sleep. Pediatrics. 2012;129(6):1091-6.

18. Touchette E, Dionne G, Forget-Dubois N, Petit D, Pérusse D, Falissard B, et al. Genetic and environmental influences on daytime and nighttime sleep duration in early childhood. Pediatrics. 2013;131(6):e1874-80.

19. Crispim JN, Boto LR, Melo IS, Ferreira R. Padrões de sono e fatores de risco para provação do sono numa população pediátrica portuguesa [Sleep pattern and risk factos for sleep deprivation in a portuguese pediatric population]. Acta Pediatr Port. 2011;42(3):93-8. Portuguese

20. Gulliford MC, Price CE, Rona RJ, Chinn S. Sleep habits and height at ages 5 to 11. Arch Dis Child. 1990;65(1):119-22.

21. Iglowstein I, Jenni OG, Molinari L, Largo RH. Sleep duration from infancy to adolescence: reference values and generational trends. Pediatrics. 2003;111(2):302-7.

22. Canet T. Sleep-wake habits in Spanish primary school children. Sleep Med. 2010;11(9):917-21.

23. Blair PS, Humphreys JS, Gingras P, Taheri S, Scott N, Emond A, et al. Childhood sleep duration and associated demographic characteristics in an English cohort. Sleep. 2012;35(3):353-60.
24. Faruqui F, Khubchandani J, Price JH, Bolyard D, Reddy R. Sleep disorders in children: a national assessment of primary care pediatrician practices and perceptions. Pediatrics. 2011;128(3):539-46.

\section{CONFLITO DE INTERESSES}

Os autores declaram não ter conflitos de interesses.

\section{COMISSÃO DE ÉTICA}

Estudo realizado após parecer favorável da Comissão de Ética para a Saúde da ARS Norte.

\section{ENDEREÇO PARA CORRESPONDÊNCIA}

Maria Adriana Rangel

Serviço Pediatria - Centro Hospitalar Vila Nova de Gaia/Espinho, EPE

Rua Francisco Sá Carneiro, 4400-129 Vila Nova de Gaia

E-mail: mariaadrianarangel@hotmail.com

Recebido em 28-02-2015

Aceite para publicação em 19-08-2015

Artigo escrito ao abrigo do novo acordo ortográfico.

\section{ABSTRACT}

\section{SLEEP QUALITY AND SLEEP DISTURBANCES IN HEALTHY CHILDREN IN GAIA:A CROSS SECTIONAL STUDY}

Objectives: To evaluate the quality of sleep and estimate the prevalence of sleep disorders in children from two to 10 years old at a health center in the metropolitan area of Porto.

Study type: Cross-sectional study.

Setting: Child health consultations at a primary health care center in the urban area of Grande Porto ACeS VII - Gaia.

Participants: Children aged from 2 to 10 years enrolled in a health center of ACeS Grande PortoVII - Gaia.

Methods: The Children's Sleep Habits Questionnaire (CSHQ-PT) translated and validated for use in Portuguese, and a questionnaire on individual, social, and family issues, were applied to children between two and 10 years of age, who attended a child health consultation between April and June, 2014.

Results: A total of 131 questionnaires were analyzed. The median age was 5 years and $55 \%$ of the subjects were in the preschool age with a predominance of male subjects (53.5\%). The Sleep Disturbance Rate (SDR) was higher in pre-school aged children $(p<0.032$ ). Using a cut off score of 41 , an elevated SDR was found in $80.2 \%$ (median 47 ). A higher SDR was associated with the need for a family member in the bedroom $(p=0.005)$ or in bed $(p<0.001)$ to help the child fall asleep, or the use of television $(p=0.006)$ or videogames $(p=0.04)$ before bedtime. Children who slept alone or shared a bedroom with a sibling had a lower SDR than those sharing bedroom with their parents $(p=0.015)$. A lower SDR was associated with higher academic performance $(p=0.029)$.

Conclusion: Sleep-disturbances are frequent in this population, particularly in younger ages. A significant association was found between the need for the presence of a relative in the room or in bed to help the child fall asleep. Television viewing and use of videogames were associated with a reduced total sleep time.

Keywords: Sleep Disorders; Epidemiologic Factors; Primary Health Care. 